\title{
The influence of haematuria on the utility of the BTA stat test in the diagnosis of bladder cancer: a prospective study
}

\section{Xiong Xiong ( $\sim$ szbzbb@163.com )}

Chongqing Medical University First Affiliated Hospital

Wei He

Chongqing Medical University First Affiliated Hospital

Bin Xiong

People's Hospital of Nanchuan

Feng Yao

Banan People's Hospital

Research article

Keywords: bladder cancer, BTA stat test, diagnostic, haematuria

Posted Date: January 15th, 2021

DOI: https://doi.org/10.21203/rs.3.rs-64771/v3

License: (a) (i) This work is licensed under a Creative Commons Attribution 4.0 International License. Read Full License 


\section{Abstract}

Background The urinary bladder tumour antigen (BTA) stat test has already been used for the diagnosis and monitoring of bladder cancer (BC). However, more evidence is needed regarding its efficacy and utility in the clinic. In this study, we investigated the influence of haematuria on the performance of the BTA stat test in a clinical cohort.

Methods Urine samples from 836 subjects, including 50 healthy volunteers, 553 patients with benign urologic disorders, 124 patients with histologically proven $\mathrm{BC}$, and 109 patients with other histologically proven urologic cancers, were analysed by the BTA stat test and urinalysis. We detected the sensitivity and specificity of the BTA stat test in each group and analysed the effect of haematuria on the specificity.

Results Our data show that $58.06 \%$ of patients in the BC group had haematuria. Haematuria with benign prostatic hyperplasia $(\mathrm{BPH})$, renal hamartoma $(\mathrm{RH})$ and urolithiasis were identified in $39.01 \%, 42.86 \%$ and $66.49 \%$ of patients with benign urologic disorders, respectively. Haematuria was identified in $48.72 \%$ of prostatic cancer patients and $67.74 \%$ of renal cancer patients. The overall sensitivity of the BTA stat test was $90.32 \%$. The sensitivity was $97.22 \%$ in BC patients with haematuria and $80.77 \%$ in $\mathrm{BC}$ patients without haematuria. The overall specificity in healthy individuals, patients with benign urologic disorders and patients with other urologic cancers was $50.84 \%$. In all patients with haematuria, the specificity of the BTA stat test was $15.82 \%$, while the specificity was $72.6 \%$ in patients without haematuria.

Conclusions Haematuria has a significant influence on the BTA stat test. The performance of the BTA stat test can be increased if patients with known or obvious haematuria conditions are excluded from the test.

\section{Background}

Bladder cancer (BC) is the ninth most common cancer disease worldwide, and the incidence is particularly high in men [1]. Early $\mathrm{BC}$ has a better prognosis, but the progression of the disease greatly increases the rates of metastasis and mortality [2]. Therefore, timely detection and diagnosis of $B C$ are essential. Currently, cystoscopy and urine cytology are the best tests for the diagnosis of BC [3]. However, there are some limitations in practical clinical applications. Cystoscopy is invasive and costly [4]. Urine cytology has ideal specificity, but has low sensitivity, and the interpretation of the results depends highly on the skill of the examiner [5]. In recent years, molecular markers have been proposed for the detection of bladder tumours. Some markers have been developed as commercial reagents. The advantages of these markers over cystoscopy and urine cytology are that these markers are simple to use and less painful and increased sensitivity. In contrast, their disadvantages are mainly suboptimal accuracy and high inter-observer variability [6]. Hence, to be widely used, the specificity and sensitivity of these molecular markers need to be tested in clinical practice to determine their suitable diagnostic conditions.

The bladder tumour antigen (BTA) stat test is the most relevant BC marker detection method and has been approved by the US Food and Drug Administration for the diagnosis and monitoring of $\mathrm{BC}[7,8]$. This antigen has been recognized as a human complement factor H-related protein (hCFHrp) that inhibits the complement pathway to cause cytolysis in cells, with a resulting selective advantage for the tumour [9]. Previous studies have suggested that the sensitivity of the BTA stat test is superior to that of urine cytology. However, more evidence is needed regarding its efficacy and utility in the clinic, especially when interference by some pathological factors may occur.

In the clinic, haematuria is a symptom of BC that cannot be ignored. The initial diagnosis of BC most often occurs after an episode of gross or microscopic haematuria. Over $80 \%$ of patients with $B C$ have some degree of haematuria $[10,11]$. However, there are many causes of haematuria other than $\mathrm{BC}$, including many benign urologic diseases, urinary tract infection, ureteric and renal stones, etc. Even between $9 \%$ and $18 \%$ of normal individuals have some degree of haematuria [12]. hCFHrp as a serum factor, haematuria maybe introduce it into urine, which can cross react with BTA stat test assays. Hence, if haematuria has a serious effect on the specificity of the BTA stat test, using this test to screen and monitor BC can lead to unnecessary investigations and anxiety. 
In order to investigate the effect of haematuria on the specificity of BTA stat test and further clarify whether BTA stat test detects tumour antibodies or serum antibodies, we designed a prospective study. This study analysed the specificity of BTA stat test in with or without haematuria urine samples from Healthy group, bladder cancer group, benign urologic diseases group and other urologic cancer group.

\section{Material And Methods}

\section{Patients}

Table 1 Patient demographics (Table 1 was uploaded as an additional file)

After receiving approval by the local institutional ethics committee and obtaining informed consent, this study prospectively collected urine samples from 1,478 patients who visited the Urology Department of the First Affiliated Hospital of Chongqing Medical University between January 2018 and December 2019. All patients who provided urine samples were numbered after urine collection, and each sample was subjected to a routine urine test and the BTA stat test. None of the participants had mechanical manipulation of the urethra or bladder within the last two weeks before specimen collection. No participants received any medical therapy (i.e., instillation therapy or systemic chemotherapy) before the collection urine samples.

Then, we collected the clinical data of the enrolled patients, who completed related inspections as needed, including a urinary ultrasound, CT, PSA screening, etc. Of these examinations, cystoscopic biopsy was performed in patients suspected of having $\mathrm{BC}$ (due to painless gross or microscopic haematuria or irritative voiding symptoms). For patients suspected of renal cancer based on CT, postoperative pathological confirmation was performed. Suspected diagnoses of prostate cancer based on the digital rectal examination and PSA test were confirmed by prostate puncture cytology or postoperative pathology. Benign diseases of the urinary system were diagnosed according to the corresponding diagnostic criteria. All diagnoses were confirmed by two senior urologists.

The participants who ultimately did not belong to one of the diagnostic categories or refused further tests to confirm the diagnosis were excluded. Except for the urinary tract infection (UTI) group in the benign urologic diseases group, patients with active UTIs in the other groups as indicated by either symptom reports or pyuria were excluded. Except for the urolithiasis group in the benign urologic diseases group, patients with a recent history of urolithiasis in the other groups were excluded.

Finally, the study group consisted of 124 patients with BC. The benign urologic diseases group consisted of benign prostatic hyperplasia $(B P H, n=141)$, renal cyst $(n=66)$, benign adrenal tumour (BAT, $n=75)$, renal hamartoma $(R H, n=28)$, urolithiasis $(n=185)$, and UTI $(n=58)$. The other urologic cancer group consisted of 78 patients with prostate cancer and 31 patients with renal cancer. According to the previous numbering, the urine routine test and BTA results of the initial test were retrieved for further analysis.

In addition, we recruited a group of healthy volunteers without any clinical symptoms from the physical examination centre during the study period. These participants underwent routine physical examinations at the physical examination centre, including a urinary occult blood test, urinary ultrasound, CT and PSA screening. Those with normal physical examination results who agreed to complete the follow-up tests were included in the study. Finally, the healthy volunteer group included 50 individuals. Their urine was also collected for a BTA stat test and urine analysis.

Fig. 1 The flow chart diagram of patient selection and classification is shown in the Figure 1 below. (Fig. 1 was uploaded as an additional file)

\section{Haematuria detection}


In our study, routine urinalysis includes a dry chemical analysis, flow cytometry analysis and microscopic examination of the urine sediment. Haematuria included gross haematuria and microhaematuria. Gross haematuria was defined as a colour change in the appearance of urine and confirmed by the demonstration of red blood cells in the urinary sediment as shown by microscopy [13]. Microhaematuria was defined as 3 or more red blood cells in the urinary sediment per highpower microscopic field [14].

\section{BTA stat test}

The BTA stat test (Polymedco, NY, USA) is a one-step qualitative assay. We assayed all samples according to the manufacturer's instructions [15]. Three drops of urine were placed on the BTA stat test device, which contains a small lateral flow immunochromatographic assay. The urine reacts with colloidal gold-conjugated anti-bladder tumour-associated antigen antibody to form an immune complex. Then, when the immune complex flows through the detection area, it binds another anti-bladder tumour-associated antigen antibody and forms a visible colour band. If there is no hCFHrp in the urine, no visible line forms in the detection area. Regardless of the presence or absence of hCFHrp in the urine sample, the control area can bind the detection antibody to form a visible line. Therefore, when both the target and control zones form two visible lines, the reading is positive; when only the control line is formed, the reading is negative. The absence of a visible control line indicates that the test is invalid and needs to be repeated.

\section{Statistical analysis}

The statistical analysis was performed using SPSS software, version 20.0 (Chicago, IL, USA). Analysis of the study population characteristics relied on descriptive statistics. Qualitative variables are expressed as proportions. Sensitivity was defined as the ratio of the number of true positive test results and the number of subjects with confirmed BC. Specificity was defined as the ratio of the number of true negative test results and the number of subjects without BC. The exact $95 \%$ confidence intervals (Cls) indicate the precision of the sensitivity and specificity [7]. Groups were compared using Fisher's exact test or the Pearson $\chi^{2}$ test. A $P$-value $<0.05$ was considered significant.

\section{Results}

\section{BTA stat test in patients with BC}

The overall sensitivity of the BTA stat test in the detection of BC was $90.32 \%$. The sensitivity of the BTA stat test was $83.87 \%$ in pTa tumours, $95.83 \%$ in pT1 tumours, and $96.43 \%$ in pT2 tumours. None of the patients with pT3-pT4 tumours had negative BTA stat test results. Regarding the histologic grades, the sensitivity was $80 \%$ in papillary urothelial neoplasm of low malignant potential (PUNLMP) tumours, $70 \%$ in low-grade tumours, and $97.75 \%$ in high-grade tumours. In the BC group, $58.06 \%$ of patients had haematuria. The sensitivity of the BTA stat test was $97.22 \%$ in BC patients with haematuria and $80.77 \%$ in $\mathrm{BC}$ patients without haematuria, and the difference was statistically significant $(P<0.05)$. (Table 2).

Table 2 Sensitivity of the BTA stat test by tumour stage and grade (Table 2 was uploaded as an additional file)

\section{BTA stat test in healthy volunteers and patients with other urologic disorders}

Among the urinary specimens from the 50 healthy individuals, none of the specimens had positive BTA stat test results. Of the 553 patients with benign urologic diseases, $52.62 \%$ of patients had false-positive BTA stat test results. The incidence of false-positive results was related to the observed condition as follows: $68.11 \%$ of patients with urolithiasis, $84.48 \%$ of patients with UTI, $43.97 \%$ of patients with BPH, $37.33 \%$ of patients with BAT and $46.43 \%$ of patients with RH had falsepositive BTA stat test results. In total, $19.7 \%$ of patients with renal cysts had false-positive BTA stat test results. In addition, among the patients with prostatic cancer and renal cancer, the false-positive rates of the BTA stat test were $55.12 \%$ and $51.61 \%$, respectively. 
The specificity of the BTA stat test was $100 \%$ if only healthy individuals were considered. The overall specificity (healthy individuals, patients with benign urologic diseases and patients with other urologic cancers) of the BTA stat test was $50.84 \%$ (Table 3 ).

Table 3 Specificity of the BTA stat test in 50 healthy subjects and patients in eight disease categories (Table 3 was uploaded as an additional file)

\section{Effect of haematuria on the BTA stat test}

Among the patients with benign urologic diseases, those with haematuria accounted for a relatively high proportion. Haematuria was present in $39.01 \%, 42.86 \%$ and $66.48 \%$ of patients with $\mathrm{BPH}, \mathrm{RH}$ and urolithiasis, respectively. Among the urologic cancers, $58.06 \%$ of the BC patients exhibited haematuria, while $48.72 \%$ of the prostatic cancer patients and $67.74 \%$ of the renal cancer patients exhibited haematuria (Fig. 2).

Haematuria had a significant effect on the specificity of the BTA stat test in each group. In the group with benign urologic diseases with haematuria, the specificity of the BTA stat test was $11.34 \%$, which was significantly lower than the $74.6 \%$ observed in the group without haematuria with the same conditions $(P<0.05)$. The specificity of this test decreased to $8.94 \%-31.58 \%$ in patients with BPH, renal cyst, BAT, RH and urolithiasis associated with haematuria. When we removed patients with haematuria from this analysis, the specificity of the BTA stat test increased to $73.21-98.08 \%$ $(P<0.05)$. Among the prostatic cancer patients with haematuria, the specificity of the BTA stat test was $28.95 \%$, while the specificity increased to $60 \%$ after removing patients with haematuria from this analysis $(P<0.05)$. In all groups of patients with haematuria, the specificity of the BTA stat test was $15.82 \%$, and the overall specificity of the BTA stat test was $70.5 \%$ in patients without haematuria. The difference was statistically significant $(P<0.05)$ (Table 4).

In addition, our study found that the specificity of the BTA stat test was correlated with the severity of haematuria. The specificity of the BTA stat test in patients with haematuria detected under a microscope was significantly higher than that in patients with gross haematuria $(P<0.05)$.

Fig. 2 The proportion of haematuria in various diseases and the positive rate of BTA in haematuria patients (Fig. 2 was uploaded as an additional file)

Figure legends

Fig. 1 a. The proportion of haematuria in patients with $\mathrm{BC}$, prostatic cancer, renal cancer and benign urologic diseases. The data are derived from 786 patients. b. The positive rate of BTA in patients with BC, prostatic cancer, renal cancer and benign urologic diseases. The data are derived from 353 patients.

Table 4 Specificity of the BTA stat test in patients with or without haematuria in eight disease categories (Table 4 was uploaded as an additional file)

Table 5 The specificity of the BTA stat test in microscopic haematuria and gross haematuria (Table 5 was uploaded as an additional file)

\section{Discussion}

Among the non-invasive urine tests, urine tests with molecular biomarkers are promising tools for the diagnosis of $\mathrm{BC}$ [16]. However, these tests are still not well established in daily clinical routine and the standard diagnostic workup of BC. Further evaluation of the usefulness of these biomarkers in complex clinical situations is needed before they are recommended for widespread clinical use. BC patients often exhibit haematuria, which is also the most common manifestation of other urological diseases $[11,12]$. Hence, it is important to evaluate the usefulness of molecular biomarkers of $\mathrm{BC}$ in the context of haematuria interference. 
The BTA stat test is a molecular urine test for BC that is currently subject to the most attention. The BTA stat test specifically recognizes bladder tumour-associated antigen (hCFHrp) in urine through monoclonal antibodies. This antigenic protein is believed to be isolated from the urine of patients with BC but cannot be detected in the urine of most healthy individuals [9]. Previous studies have compared the sensitivity of the BTA stat test and cytology detection in patients with BC diagnosed by cystoscopy, and the sensitivity of the BTA stat test was found to be superior to that of urine cytology and bladder irrigation cytology [17-19]. However, data regarding its effectiveness in real-world clinical situations are limited. Specifically, its specificity under haematuria interference situations needs further observations. In this study, we investigated the effect of haematuria on the sensitivity and specificity of the BTA stat test in a clinical cohort.

Previous studies have shown that the sensitivity of the BTA stat test ranges between $57 \%$ and $83 \%$ [20]. In our study, the sensitivity of the BTA stat test in detecting patients without haematuria BC was $80.77 \%$, which is consistent with previous studies. When we analysed only patients with haematuria, the overall sensitivity of the BTA stat test was $97.22 \%$. This finding illustrates the effect of haematuria on its sensitivity. Because of false-positives, haematuria patients may exhibit higher sensitivity. Our research shows that haematuria had a significant effect on the specificity of the BTA stat test in each group. In non-BC with haematuria patients, the specificity of the BTA stat test was significantly lower than that in patients without haematuria who had the same conditions (the data show that except for the UTI and renal cancer groups, the reasons may be that urinary tract infections also significantly affect the specificity of the BTA stat test and that the sample size of the renal carcinoma group was too small). Furthermore, compared with other studies, our study showed a lower specificity. In a multicentre US study, the specificity of the BTA stat test in patients with BPH, urolithiasis and UTI was $88.5 \%$, $50 \%$ and $76 \%$, respectively [21]. In this study, the specificity of the BTA stat test decreased to only $31.89 \%$ and $15.52 \%$ in patients with urolithiasis and UTI, respectively. In patients with prostatic cancer and renal cancer, the specificity of the BTA stat test was only $44.87 \%$ and $48.39 \%$, respectively, which may be because we did not exclude patients with haematuria from our total enrolment in these data. In addition, our study found that the specificity of the BTA stat test was correlated with the severity of haematuria. The specificity of the BTA stat test in patients with haematuria detected under a microscope was significantly higher than that in patients with gross haematuria.

Our results suggest that haematuria leads to high positive rates. Considering that hCFHrp is a serum factor, the BTA stat test can possibly concomitantly detect serum proteins that cause haematuria. A study supports our conclusion; the Makito Miyake group built an experimental haematuria model. These authors added whole blood to BTA-negative urine samples and found that spiking BTA-negative urine samples with as little as $1 \mu \mathrm{l}$ whole blood/10 $\mathrm{ml}$ urine was enough to produce a positive BTA stat test result [22]. These studies confirm that the BTA stat test is very unsatisfactory for the diagnosis of $\mathrm{BC}$. On the one hand, most patients with $\mathrm{BC}$ present with haematuria, and gross or microscopic haematuria is often the first symptom in $\mathrm{BC}$ patients $[11,12]$. On the other hand, haematuria is the most common symptom in urology patients. In many benign or tumorous urologic diseases, haematuria is often the only manifestation [23]. Even $9 \%$ to $18 \%$ of normal individuals have some degree of haematuria [12]. This fact indicates that the BTA stat test may be prone to detect particularly high false-positives in haematuria patients.

We analysed patients with haematuria symptoms in a clinical setting and found that the specificity of the BTA stat test was only $15.82 \%$ in all groups of patients with haematuria. These data indicate that when only patients with haematuria symptoms who were suspected of having BC were considered, the specificity of diagnosing BC with the BTA stat test was only $15 \%$. In addition, the proportion of BC patients with haematuria is very small, especially patients with microscopic haematuria. Previous studies have shown that only approximately $1 \%$ of patients with microscopic haematuria actually have BC $[10,24,25]$. In this case, if the BTA stat test has such a high false-positive rate for haematuria, this test lacks further directivity, and its clinical utility and benefit are low. The cost of screening includes the cost of labelling each patient and the cost of evaluating patients with false-positive results. Adding any test to the clinical assessment increases the costs; thus, the cost-effectiveness needs to be balanced by the benefits. 
Optimal screening strategies require not only identifying a method with high sensitivity in a population with significant disease prevalence but also a method with reasonable specificity [26]. The specificity of the marker plays a key role because patients with false-positive results require additional "unnecessary" tests. Our results suggest that the BTA stat test cannot achieve ideal effective specificity and that using this test to screen and monitor BC may lead to "unnecessary" costs. Therefore, the BTA stat test is not recommended for screening or diagnosing BC in patients with haematuria.

In addition, in the case of follow up of urinary tumour markers, a negative predictive value is also very important if the goal is to avoid a more invasive cystoscopy based on a urinary tumour marker report. In our study, the negative predictive value of the BTA stat test was $97.09 \%$, which is similar to the results reported in previous studies. Our study also shows that the negative predictive value of the BTA in patients with haematuria was significantly higher than that in patients without haematuria, and the difference was statistically significant $(P<0.05)$. Nevertheless, notably, because the negative predictive value is affected by the prevalence, even if the diagnostic performance is very poor, this value can be as high as $90 \%$ or greater when the prevalence is relatively low. Currently, it is not our objective to use the negative predictive value to measure the diagnostic value of diagnostic experiments. After identifying high-risk groups, the negative predictive value can play its greatest role, but this requires large-scale prospective studies to determine the actual prevalence in high-risk groups. If the prevalence is still low in high-risk groups, the cost and actual benefits of screening need to be considered. Further studies can compare and analyse the specific costs involved in the clinical practice of qualitative urinary tract testing. However, there are no available data related to this problem.

Various biomarkers have been developed for the evaluation of $\mathrm{BC}$, including protein-based and gene-based biomarkers. Similar to BTA, the use of the protein marker NMP22 is also hampered by haematuria [27-28]. Notably, these results do not indicate that the high false-positive rate of protein-based makers in patients with haematuria is directly caused by red blood cells in the urine. Other factors that promote haematuria may also promote tumour cell shedding, thereby increasing the positive detection rate of these makers. Hence, protein-based biomarkers need to be tested under specific circumstances. The gene-based detection of $\mathrm{BC}$, such as the UroVysio test and Cxbladder test, performs better under haematuria interference [29-30]. However, these testing methods are far more complex, and their high cost is the greatest obstacle to their widespread use.

There are some limitations in our study. The numbers of patients in the T3, T4, and undifferentiated cancer groups are low. The sensitivity of $100 \%$ in these patient groups may not be statistically significant. The sample size in the renal cancer group is small, which may also cause some bias. We provide and retain these data to allow readers to evaluate the sample size and clinical data.

\section{Conclusions}

Our research shows that the specificity of the BTA stat test is very low under haematuria conditions; thus, this test should not be used for the diagnosis of BC in patients with haematuria. If patients with known or obvious haAematuria, urinary stones, and urinary tract infections were excluded, the application of BTA stat test will be more reliable.

\section{Abbreviations}

BTA: bladder tumour antigen

BC: bladder cancer

$\mathrm{BPH}$ : benign prostatic hyperplasia

RH: renal hamartoma

hCFHrp: human complement factor H-related protein 
UTI: urinary tract infection

BAT: benign adrenal tumour

Cl: confidence interval

TCC: transitional cell carcinoma

SCC: squamous cell carcinoma

PUNLMP: papillary urothelial neoplasm of low malignant potential

\section{Declarations}

Ethics approval and consent to participate

The procedures used in this study adhere to the tenets of the Declaration of Helsinki. Our study protocol was approved by The Ethics Committee of The First Affiliated Hospital of The Chongqing Medical University (Approval number:2017-180). Informed consent was obtained from all patients prior to inclusion.

\section{Consent for publication}

Not applicable.

\section{Availability of data and materials}

The datasets analyzed during the current study is available from the corresponding author on reasonable request.

\section{Competing interests}

The authors declare that they have no conflict of interest.

\section{Funding}

Not applicable.

\section{Authors' contributions}

XX was involved in data collection and data analysis and wrote the manuscript. WH was involved data collection and edited the manuscript. FY and BX were involved in data collection. All authors reviewed the manuscript.

\section{Acknowledgements}

Not applicable.

\section{References}

1. Antoni S, Ferlay J, Soerjomataram I, Znaor A, Jemal A, Bray F: Bladder Cancer Incidence and Mortality: A Global Overview and Recent Trends. EUR UROL 2017, 71(1):96-108

2. Abel PD: Prognostic indices in transitional cell carcinoma of the bladder. Br J Urol 1988, 62(2):103-109.

3. Babjuk M, Bohle A, Burger M, Capoun O, Cohen D, Comperat EM, Hernandez V, Kaasinen E, Palou J, Roupret M et al: EAU Guidelines on Non-Muscle-invasive Urothelial Carcinoma of the Bladder: Update 2016. EUR UROL 2017, 71(3):447-461. 
4. Sievert, K.D., et al., Economic aspects of bladder cancer: what are the benefits and costs? World J Urol, 2009. 27(3): p. 295-300.

5. Yafi FA, Brimo F, Auger M, Aprikian A, Tanguay S, Kassouf W: Is the performance of urinary cytology as high as reported historically? A contemporary analysis in the detection and surveillance of bladder cancer. Urol Oncol 2014, 32(1):21-27.

6. Vrooman, O.P.J. and J.A. Witjes, Urinary markers in bladder cancer. European Urology, 2008. 53(5): p. 909-916.

7. Tsui KH, Chen SM, Wang TM, Juang HH, Chen CL, Sun GH, Chang PL: Comparisons of voided urine cytology, nuclear matrix protein-22 and bladder tumor associated antigen tests for bladder cancer of geriatric male patients in Taiwan, China. ASIAN J ANDROL 2007, 9(5):711-715.

8. Chen A, Fu G, Xu Z, Sun Y, Chen X, Cheng KS, Neoh KH, Tang Z, Chen S, Liu M et al: Detection of Urothelial Bladder Carcinoma via Microfluidic Immunoassay and Single-Cell DNA Copy-Number Alteration Analysis of Captured UrinaryExfoliated Tumor Cells. CANCER RES 2018, 78(14):4073-4085.

9. Heicappell R, Muller M, Fimmers R, Miller K: Qualitative determination of urinary human complement factor H-related protein (hcfHrp) in patients with bladder cancer, healthy controls, and patients with benign urologic disease. UROL INT 2000, 65(4):181-184.

10. Messing EM, Young TB, Hunt VB, Roecker EB, Vaillancourt AM, Hisgen WJ, Greenberg EB, Kuglitsch ME, Wegenke JD: Home screening for hematuria: results of a multiclinic study. J Urol 1992, 148(2 Pt 1):289-292.

11. Messing EM, Young TB, Hunt VB, Newton MA, Bram LL, Vaillancourt A, Hisgen WJ, Greenberg EB, Kuglitsch ME, Wegenke JD: Hematuria home screening: repeat testing results. J Urol 1995, 154(1):57-61.

12. Grossfeld GD, Litwin MS, Wolf JS, Hricak H, Shuler CL, Agerter DC, Carroll PR: Evaluation of asymptomatic microscopic hematuria in adults: the American Urological Association best practice policy-part l: definition, detection, prevalence, and etiology. UROLOGY 2001, 57(4):599-603.

13. Bolenz, C., et al., The Investigation of Hematuria. Dtsch Arztebl Int, 2018. 115(48): p. 801-807.

14. Horstmann, M., T. Franiel and M.O. Grimm, Differential diagnosis of hematuria. Der Urologe, 2014. 53(8): p. $1215-1226$.

15. Pode D, Shapiro A, Wald M, Nativ O, Laufer M, Kaver I: Noninvasive detection of bladder cancer with the BTA stat test. J Urol 1999, 161(2):443-446.

16. Lodewijk I, Duenas M, Rubio C, Munera-Maravilla E, Segovia C, Bernardini A, Teijeira A, Paramio JM, Suarez-Cabrera C: Liquid Biopsy Biomarkers in Bladder Cancer: A Current Need for Patient Diagnosis and Monitoring. INT J MOL SCl $2018,19(9)$.

17. Ellis WJ, Blumenstein BA, Ishak LM, Enfield DL: Clinical evaluation of the BTA TRAK assay and comparison to voided urine cytology and the Bard BTA test in patients with recurrent bladder tumors. The Multi Center Study Group. UROLOGY 1997, 50(6):882-887.

18. Ramakumar S, Bhuiyan J, Besse JA, Roberts SG, Wollan PC, Blute ML, O'Kane DJ: Comparison of screening methods in the detection of bladder cancer. J Urol 1999, 161(2):388-394.

19. D'Hallewin MA, Baert L: Initial evaluation of the bladder tumor antigen test in superficial bladder cancer. J Urol 1996, 155(2):475-476.

20. Noon, A.P. and A.R. Zlotta, Urothelial Bladder Cancer Urinary Biomarkers. EJIFCC, 2014. 25(1): p. 99-114.

21. Sarosdy MF, Hudson MA, Ellis WJ, Soloway MS, DeVere WR, Sheinfeld J, Jarowenko MV, Schellhammer PF, Schervish EW, Patel JV et al: Improved detection of recurrent bladder cancer using the Bard BTA stat Test. UROLOGY 1997, 50(3):349-353.

22. Miyake M, Goodison S, Rizwani W, Ross S, Bart GH, Rosser CJ: Urinary BTA: indicator of bladder cancer or of hematuria. WORLD J UROL 2012, 30(6):869-873.

23. Avellino GJ, Bose S, Wang DS: Diagnosis and Management of Hematuria. Surg Clin North Am 2016, 96(3):503-515.

24. Britton JP, Dowell AC, Whelan P, Harris CM: A community study of bladder cancer screening by the detection of occult urinary bleeding. J Urol 1992, 148(3):788-790. 
25. Murakami S, Igarashi T, Hara S, Shimazaki J: Strategies for asymptomatic microscopic hematuria: a prospective study of 1,034 patients. J Urol 1990, 144(1):99-101.

26. Lotan Y, Elias K, Svatek RS, Bagrodia A, Nuss G, Moran B, Sagalowsky Al: Bladder cancer screening in a high risk asymptomatic population using a point of care urine based protein tumor marker. J Urol 2009, 182(1):52-57, 58.

27. Miyake, M., et al., Influencing factors on the NMP-22 urine assay: an experimental model. BMC Urol, 2012. 12: p. 23.

28. Huber, S., et al., Nuclear matrix protein-22: a prospective evaluation in a population at risk for bladder cancer. Results from the UroScreen study. BJU Int, 2012. 110(5): p. 699-708.

29. Todenhöfer, T., et al., Impact of different grades of microscopic hematuria on the performance of urine-based markers for the detection of urothelial carcinoma. Urol Oncol, 2013. 31(7): p. 1148-54.

30. Kavalieris, L., et al., A segregation index combining phenotypic (clinical characteristics) and genotypic (gene expression) biomarkers from a urine sample to triage out patients presenting with hematuria who have a low probability of urothelial carcinoma. BMC Urol, 2015. 15: p. 23.

\section{Tables}

Table 1

Patient demographics

\begin{tabular}{|llllllll|}
\hline Group & & Total & Male & Female & Mean age & Range age \\
\hline Bladder cancer & & 124 & 103 & 21 & 66.7 & $26-89$ \\
\hline Healthy volunteers & & 50 & 30 & 20 & 50.2 & $25-72$ \\
\hline Benign urologic diseases & 553 & 378 & 175 & 62.4 & $22-95$ \\
\hline & BPH & 141 & 141 & 0 & 70.7 & $51-95$ \\
\hline & Renal cyst & 66 & 36 & 30 & 64.1 & $47-87$ \\
\hline & BAT & 75 & 27 & 48 & 53.3 & $22-74$ \\
\hline & RH & 28 & 6 & 22 & 55 & $31-70$ \\
\hline & Urolithiasis & 185 & 129 & 56 & 60 & $26-90$ \\
\hline & UTI & 58 & 39 & 19 & 63.3 & $48-88$ \\
\hline Other urologic cancers & & 109 & 101 & 8 & 68.9 & $35-93$ \\
\hline & Prostatic cancer & 78 & 78 & 0 & 72 & $52-93$ \\
\hline & Renal cancer & 31 & 23 & 8 & 61 & $35-76$ \\
\hline BPH, benign prostatic hyperplasia; BAT, benign adrenal tumour; RH, renal hamartoma; UTI, urinary tract infection \\
\hline
\end{tabular}

Table 2 Sensitivity of the BTA stat test by tumour stage and grade 


\begin{tabular}{lcclcc}
\hline Variable & Negative & Positive & Total & Sensitivity (\%) & 95\% CI \\
\hline Bladder cancer & 12 & 112 & 124 & 90.32 & $83.36-94.68$ \\
Pathologic subtype & & & & & \\
\hline TCC & 11 & 107 & $118(95.16 \%)$ & 90.68 & $83.57-95.02$ \\
SCC & 1 & 4 & $5(4.03 \%)$ & 80 & $29.88-98.95$ \\
$\quad$ Undifferentiated carcinoma & 0 & 1 & $1(0.81 \%)$ & 100 & $5.46-100$ \\
\hline Grade & & & & & \\
\hline & 1 & 4 & $5(4.03 \%)$ & 80 & $29.88-98.95$ \\
\hline PUNLMP & 9 & 21 & $30(24.19 \%)$ & 70 & $50.44-84.59$ \\
\hline Low-grade & 2 & 87 & $89(71.77 \%)$ & 97.75 & $91.35-99.61$ \\
\hline
\end{tabular}

\section{Pathologic T stage}

\begin{tabular}{cccccc}
\hline Ta & 10 & 52 & $62(50 \%)$ & 83.87 & $71.87-91.59$ \\
\hline T1 & 1 & 23 & $24(19.35 \%)$ & 95.83 & $76.88-99.78$ \\
\hline T2 & 1 & 27 & $28(22.58 \%)$ & 96.43 & $79.76-99.81$ \\
\hline T3 & 0 & 4 & $4(3.23 \%)$ & 100 & $39.57-100$ \\
\hline T4 & 0 & 6 & $6(4.84 \%)$ & 100 & $51.68-100$
\end{tabular}

\section{Urine analysis}

\begin{tabular}{lcrrrr}
\hline Haematuria & 2 & 70 & $72(58.06 \%)$ & $97.22 *$ & $89.42-99.52$ \\
\hline No haematuria & 10 & 42 & $52(41.94 \%)$ & 80.77 & $67.03-89.92$ \\
\hline Statistical analysis & & & & $* P \square 0.002$ vs No haematuria \\
\hline
\end{tabular}

TCC, transitional cell carcinoma; SCC, squamous-cell carcinoma; PUNLMP, papillary urothelial neoplasm of low malignant potential; CI, confidence interval 
Table 3

Specificity of the BTA stat test in 50 healthy subjects and patients in eight disease categories

\begin{tabular}{|lcclll|}
\hline Category & BTA-negative & BTA-positive & Total & Specificity (\%) & $95 \%$ Cl \\
\hline Healthy volunteers & 50 & 0 & 50 & 100 & $91.11-100$ \\
\hline Benign urologic diseases & 262 & 291 & 553 & 47.38 & $43.16-51.63$ \\
\hline BPH & 79 & 62 & 141 & 56.03 & $47.43-64.29$ \\
\hline Renal cyst & 53 & 13 & 66 & 80.3 & $68.32-88.7$ \\
\hline BAT & 47 & 28 & 75 & 62.67 & $50.69-73.34$ \\
\hline RH & 15 & 13 & 28 & 53.57 & $34.21-71.99$ \\
\hline Urolithiasis & 59 & 126 & 185 & 31.89 & $25.35-39.2$ \\
\hline UTI & 9 & 49 & 58 & 15.52 & $7.77-27.93$ \\
\hline Other urologic cancers & 50 & 59 & 109 & 45.87 & $36.38-55.66$ \\
\hline Prostatic cancer & 35 & 43 & 78 & 44.87 & $33.74-56.51$ \\
\hline Renal cancer & 15 & 16 & 31 & 48.39 & $30.56-66.60$ \\
\hline Total & 362 & 350 & 712 & 50.84 & $47.11-54.57$ \\
\hline \begin{tabular}{l} 
BPH, benign prostatic hyperplasia; BAT, benign adrenal tumour; RH, renal hamartoma; UTI, urinary tract infection; Cl, \\
\hline confidence interval.
\end{tabular} & & & & \\
\hline
\end{tabular}

Table 4 Specificity of the BTA stat test in patients with or without haematuria symptom in eight disease categories 


\begin{tabular}{|c|c|c|c|c|c|c|c|c|c|}
\hline \multirow[t]{2}{*}{ Categories } & \multicolumn{4}{|c|}{ Haematuria } & \multicolumn{4}{|c|}{ No haematuria } & \multirow{2}{*}{$\begin{array}{c}\text { Statistical } \\
\text { analysis* } \\
P \text { value }\end{array}$} \\
\hline & Total & $\begin{array}{c}\text { BTA- } \\
\text { positive }\end{array}$ & $\begin{array}{c}\text { BTA- } \\
\text { negative }\end{array}$ & $\begin{array}{c}\text { BTA stat } \\
\text { test } \\
\text { Specificity } \\
\text { (95\% CI) }\end{array}$ & Total & $\begin{array}{c}\text { BTA- } \\
\text { positive }\end{array}$ & $\begin{array}{c}\text { BTA- } \\
\text { negative }\end{array}$ & $\begin{array}{c}\text { BTA stat } \\
\text { test } \\
\text { Specificity } \\
(95 \% \mathrm{CI})\end{array}$ & \\
\hline $\begin{array}{l}\text { Benign urologic } \\
\text { diseases }\end{array}$ & 238 & 211 & 27 & $\begin{array}{c}11.34 \% \\
(7.74- \\
16.24)\end{array}$ & 315 & 80 & 235 & $\begin{array}{l}74.6 \% \\
(69.35- \\
79.24)\end{array}$ & $\square 0.001$ \\
\hline $\mathrm{BPH}$ & 55 & 50 & 5 & $\begin{array}{c}9.09 \% \\
(3.4- \\
20.71)\end{array}$ & 86 & 12 & 74 & $\begin{array}{c}86.05 \% \\
(76.50- \\
92.27)\end{array}$ & $\square 0.001$ \\
\hline Renal cyst & 14 & 12 & 2 & $\begin{array}{c}14.29 \% \\
(2.51- \\
43.85)\end{array}$ & 52 & 1 & 51 & $\begin{array}{c}98.08 \% \\
(88.42- \\
99.9)\end{array}$ & $\square 0.001$ \\
\hline BAT & 19 & 13 & 6 & $\begin{array}{c}31.58 \% \\
(13.56- \\
56.50)\end{array}$ & 56 & 15 & 41 & $\begin{array}{l}73.21 \% \\
(59.46- \\
83.77)\end{array}$ & 0.001 \\
\hline $\mathrm{RH}$ & 12 & 10 & 2 & $\begin{array}{c}16.67 \% \\
(2.94- \\
49.12)\end{array}$ & 16 & 3 & 13 & $\begin{array}{l}81.25 \% \\
(53.69- \\
95.03)\end{array}$ & 0.001 \\
\hline Urolithiasis & 123 & 112 & 11 & $\begin{array}{c}8.94 \% \\
(4.78- \\
15.49)\end{array}$ & 62 & 14 & 48 & $\begin{array}{l}77.42 \% \\
(64.72- \\
86.68)\end{array}$ & $\square 0.001$ \\
\hline UTI & 15 & 14 & 1 & $\begin{array}{c}6.67 \% \\
(0.35- \\
33.97)\end{array}$ & 43 & 35 & 8 & $\begin{array}{c}18.6 \% \\
(8.92- \\
33.92)\end{array}$ & 0.272 \\
\hline $\begin{array}{l}\text { Other urologic } \\
\text { cancers }\end{array}$ & 59 & 39 & 20 & $\begin{array}{l}33.9 \% \\
(22.41- \\
47.49)\end{array}$ & 50 & 20 & 30 & $\begin{array}{c}60 \% \\
(45.2- \\
73.27)\end{array}$ & 0.006 \\
\hline $\begin{array}{l}\text { Prostate } \\
\text { cancer }\end{array}$ & 38 & 27 & 11 & $\begin{array}{c}28.95 \% \\
(15.99- \\
46.11)\end{array}$ & 40 & 16 & 24 & $\begin{array}{c}60 \% \\
(43.39- \\
74.72)\end{array}$ & 0.006 \\
\hline $\begin{array}{l}\text { Renal } \\
\text { cancer }\end{array}$ & 21 & 12 & 9 & $\begin{array}{c}42.86 \% \\
(22.59- \\
65.56)\end{array}$ & 10 & 4 & 6 & $\begin{array}{c}60 \% \\
(27.37- \\
86.31)\end{array}$ & 0.458 \\
\hline Total & 297 & 250 & 47 & $\begin{array}{c}15.82 \% \\
(11.96- \\
20.59)\end{array}$ & 365 & 100 & 265 & $\begin{array}{c}72.6 \% \\
(67.67- \\
77.05)\end{array}$ & $\square 0.001$ \\
\hline
\end{tabular}


$\mathrm{BPH}$, benign prostatic hyperplasia; $\mathrm{RH}$, renal hamartoma; UTI, urinary tract infection; BAT, benign adrenal tumour; CI, confidence interval; * Specificity of BTA stat test in Haematuria vs no haematuria patients.

Table 5 The specificity of the BTA stat test in microscopic haematuria and gross haematuria conditions

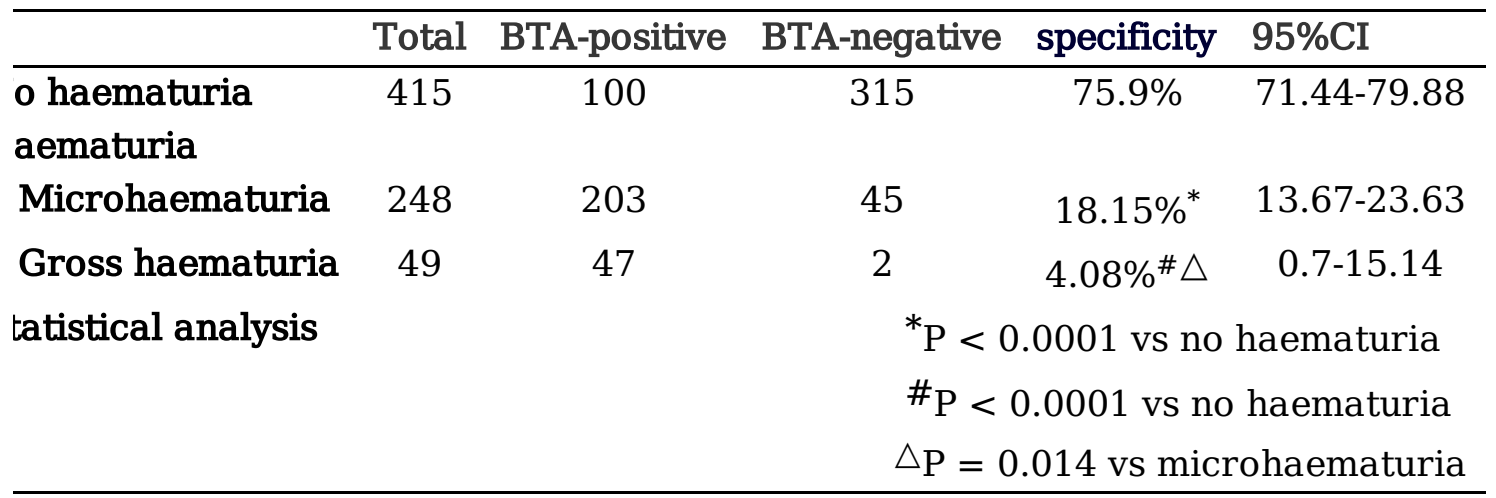

\section{Figures}




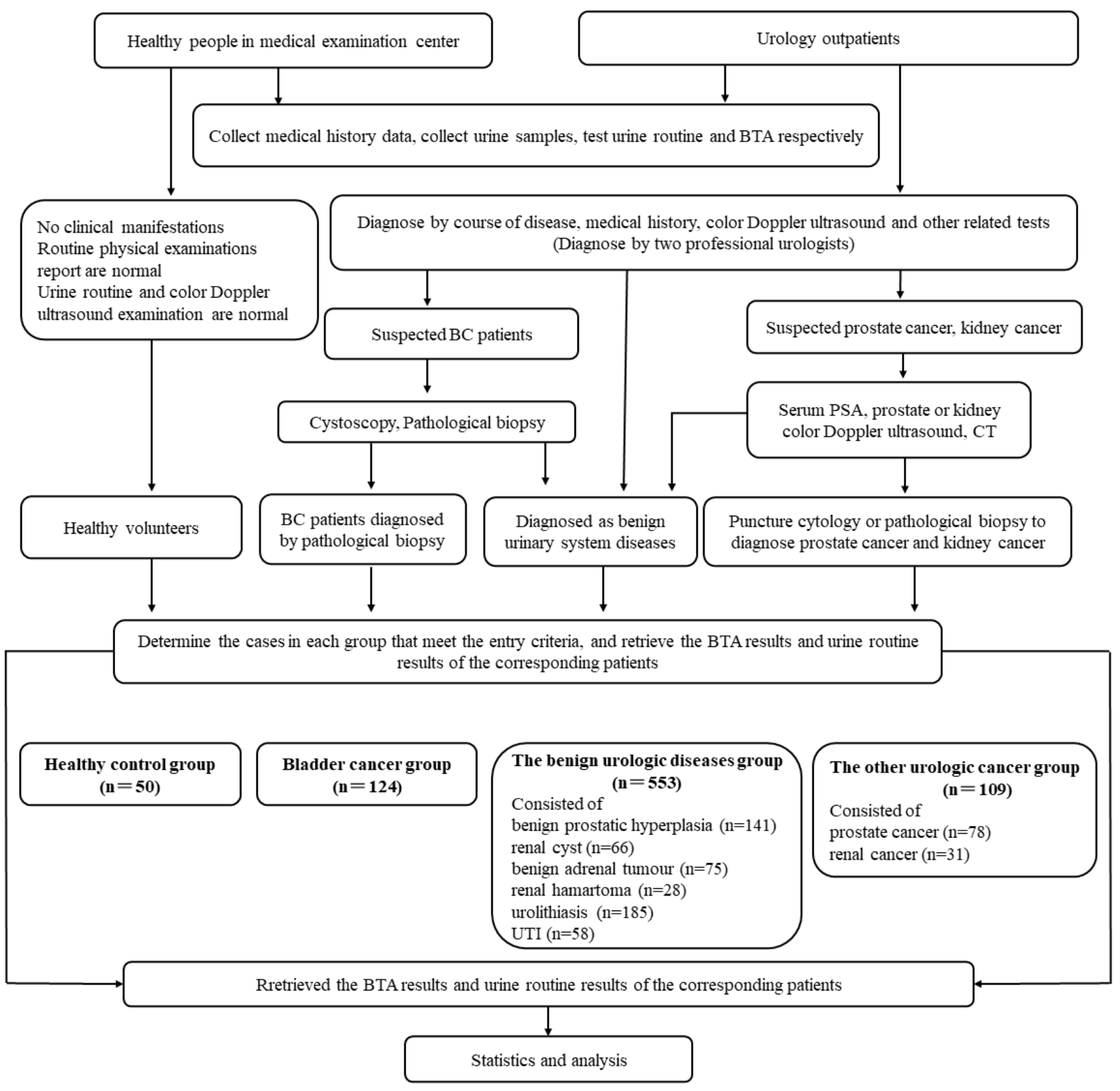

Figure 1

The flow chart diagram of patient selection and classification 

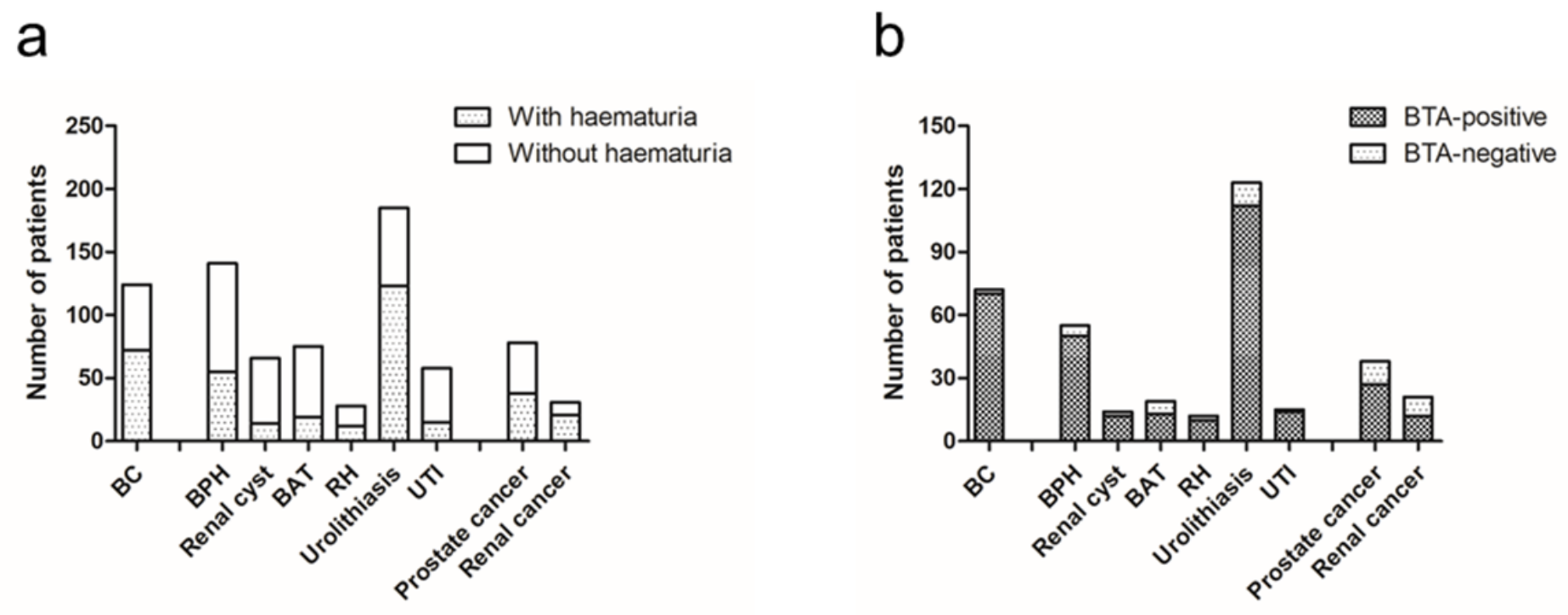

Figure 2

The proportion of haematuria in various diseases and the positive rate of BTA in haematuria patients. a. The proportion of haematuria in patients with $\mathrm{BC}$, prostatic cancer, renal cancer and benign urologic disorders. Data are from 786 patients. $b$. The positive rate of BTA in patients with BC, prostatic cancer, renal cancer and benign urologic disorders. Data are from 353 patients. 